\title{
The Ellipticity Measurements Determination of the Size of Superparamagnetic Core of Magnetic Metal Nanoparticles
}

\author{
A. Grempka ${ }^{a, b}$, M. WóJcik ${ }^{b}$, B. Piętka ${ }^{a}$, J. Szczytko $^{a, *}$
}

${ }^{a}$ Faculty of Physics, University of Warsaw, L. Pasteura 5, 02-093 Warsaw, Poland

${ }^{b}$ Faculty of Chemistry, University of Warsaw, L. Pasteura 1, 02-093 Warsaw, Poland

(Received December 05, 2019; revised version January 01, 2020; in final form January 27, 2020)

Ferrofluids, i.e., suspensions of magnetic nanoparticles, find numerous applications in biomedicine, catalysis, and optical technologies. In this work we introduce a novel and easy-to-handle way of measuring the diameter of the unoxidised core using the Faraday effect and the induced ellipticity of the light. The effects are based on the optical activity of material induced by magnetic field parallel to the direction of the propagation of the laser beam. For the spherical superparamagnetic inclusions (nanoparticles) suspended in a diamagnetic medium a theoretical model is applied. It enables one to determine the magnetic momentum of a single nanoparticle, and thus its diameter that is in coincidence with the statistical analysis of transmission electron microscopy images.

DOI: $10.12693 /$ APhysPolA.137.417

PACS/topics: 78.20.Ls, 78.67.-n, 75.50.Mm, 75.50.Cc, 61.46.+w

\section{Introduction}

Cobalt nanoparticles have attracted a great interest due to their magnetic properties. They find applications in biomedicine (drug delivery, hyperthermia, MRI contrast) [1] and catalysis [2]. Suspensions of magnetic nanoparticles called ferrofluids might be used in optical technologies [3]. Such nanoparticles have to be stable, which means that their properties should not change in time. In order to do this, nanoparticles are covered with different types of coatings including silica, surfactant, polymer, precious metal, and carbon [4].

Another way to protect metal nanoparticles is to fabricate a layer of metal oxide around the nanoparticle. The control of the thickness of the oxide shell is crucial for many applications. Importantly, in order to coat the metal nanoparticle in silica, one should first make the surface "vitreophilic" [5], that is providing $\mathrm{OH}$ groups on the metal surface. It is possible by gentle oxidation developed by Bönnemann et al. [6]. Also Peng et al. achieved the oxidation by exposing the nanoparticles to oxygen before a deposition on substrate [7].

In this work we present a simple method of determining the size of the magnetic core of nanoparticles by measuring optical activity induced by magnetic field along the direction of the propagation of the laser beam (the Faraday effect) or the ellipticity induced by the magnetic field. We compare the result with a statistical analysis of transmission electron microscopy (TEM) images.

\footnotetext{
*corresponding author; e-mail: jacek.szczytko@fuw.edu.pl
}

\section{Theoretical model}

The Faraday effect is optical activity induced by external magnetic field along the direction of propagation of the light for a certain medium. The polarization plane of the light is rotated by the Faraday rotation angle $\theta_{\mathrm{F}}$, which is a linear function of the magnetic field, i.e., $\theta_{\mathrm{F}}=V d B$, where $d$ is the length of the medium and $V$ is the Verdet constant depending on the material, temperature, and the wavelength.

In case of dilute suspension of non-interacting superparamagnetic single-domain spherical metal nanoparticles with magnetic momentum, the dependence is not linear. In fact, it can be expressed as follows [8]:

$$
\begin{aligned}
& \theta_{F}=\frac{1}{2} \operatorname{Re}\left[k_{+}-k_{-}\right]= \\
& \quad f K(\omega)\left[\frac{B_{\mathrm{ext}}}{B_{m}}\left(1-\frac{2 L(x)}{x}\right)+L(x)\right],
\end{aligned}
$$

where $B_{m}$ is the internal magnetic flux density caused by ferromagnetic magnetization, $m^{*}$ is the effective mass of the electron, $e$ is charge of the electron, $f$ is the filling factor (the volume fraction), and $L(x)=\operatorname{coth} x-\frac{1}{x}$ is the Langevin function, where $x=\frac{\mu}{k_{\mathrm{B}}, T} B_{\mathrm{ext}}$.

Assuming narrow distribution of size, from fitting of $\frac{\mu}{k_{\mathrm{B}} T}$, a magnetic momentum of a single nanoparticle can be obtained. Therefore, knowing the magnetic momentum per cobalt atom, we can determine the size of such spherical nanoparticle $K(\omega)$, through relation

$$
K(\omega)=\frac{\left(\omega_{p}^{*} \tau\right)^{2}}{2} \frac{9 \varepsilon_{h}^{3 / 2}}{1+2 \varepsilon_{h}} \frac{1-\left(1-\frac{\omega_{p}^{* 2}}{\omega^{2}}\right)^{2}(\omega \tau)^{2}}{\left[1+\left(1-\frac{\omega_{p}^{* 2}}{\omega^{2}}\right)^{2}(\omega \tau)^{2}\right]^{2}},
$$


where $\omega$ is the frequency of light, $\varepsilon_{h}$ is dielectric permittivity of the host, $\tau$ is the lifetime of momentum relaxation, and $\omega_{p}^{*}=\omega_{p} / \sqrt{1+\varepsilon_{h}}$ is the surface plasmon frequency of a spherical particle. Quantities $\varepsilon_{h}, \omega_{p}$, and $\tau$ are easily found in literature [8].

In this work we calculated the dependence of the ellipticity $\eta$ as a function of the magnetic field. It is expressed as

$$
\eta=\frac{1}{2} \operatorname{Im}\left(k_{+}-k_{-}\right)=\frac{1}{2} \operatorname{Im}\left(\frac{k_{0} A^{\text {eff }}}{\sqrt{\varepsilon_{1}^{\text {eff }}}}\right) .
$$

In the above expression $A^{\text {eff }}$ is an element of the effective dielectric tensor for magnetic nanoparticles in a dielectric host [8], and $\varepsilon_{1}^{\text {eff }}$ is assumed to be equal to $\varepsilon_{h}$. The calculated ellipticity then takes the form

$$
\eta=\frac{e B_{m}}{m^{*} c} f J(\omega)\left[\frac{B_{\text {ext }}}{B_{m}}\left(1-\frac{2 L(x)}{x}\right)+L(x)\right] .
$$

This dependence is similar to the Faraday rotation angle, while the difference is visible in the coefficient $J(\omega)$ :

$$
J(\omega)=\frac{\left(\omega_{p}^{*} \tau\right)^{2}}{2} \frac{9 \varepsilon_{h}^{3 / 2}}{1+2 \varepsilon_{h}} \frac{\left(1-\frac{\omega_{p}^{* 2}}{\omega^{2}}\right)^{2}(\omega \tau)^{3}}{\left[1+\left(1-\frac{\omega_{p}^{* 2}}{\omega^{2}}\right)^{2}(\omega \tau)^{2}\right]^{2}}
$$

\section{Experimental setup}

\subsection{Synthesis of the nanoparticles}

The cobalt nanoparticles were synthesized via thermal decomposition of dicobalt octacarbonyl, as described by Yang et al. [9]. Two samples were prepared for this experiment. Firstly, $4 \mathrm{ml}$ of o-dichlorobenzene (solvent) were placed in a three-neck-flask and stirred mechanically under inert (nitrogen) atmosphere and vacuum subsequently. This step was made to dispose of the remaining oxygen dissolved in the solvent, which might oxidise cobalt at zero degree of oxidation. Subsequently, $2 \mathrm{mmol}$ of oleic acid (OA) and $2 \mathrm{mmol}$ of triphenylphosphine (TPP) were added as surfactants for sample 1, and $3 \mathrm{mmol}$ of $\mathrm{OA}$ and $2 \mathrm{mmol}$ of trioctylphosphane oxide (TOPO) for sample 2. Then, the solvent with surfactants was heated up to $180^{\circ} \mathrm{C}$ (the boiling point of o-dichlorobenzene) and $2 \mathrm{mmol}$ of $\mathrm{Co}_{2}(\mathrm{CO})_{8}$, previously dissolved in $5 \mathrm{ml}$ of the solvent, were injected into the three-neck-flask. Huge amounts of gases and vapour were produced just after the injection. The reaction mixture was heated for another $20 \mathrm{~min}$, and after that time the reaction was quenched.

In order to purify the nanoparticles, they were centrifuged and then the ethanol was added until the first visible precipitation appeared. The surface of the cobalt nanoparticles is covered with non-polar groups of the surfactants, so the addition of a polar solvent such as methanol, ethanol or acetone causes the nanoparticles to precipitate. After the centrifugation, the remaining solvent was disposed and the nanoparticles were dissolved in a small amount of toluene. The procedure of adding ethanol and the centrifugation was repeated. The precipitated nanoparticles were again dissolved in a small amount of toluene.

\subsection{The measurement of the Faraday effect and the ellipticity}

The source of the monochromatic light was a $\mathrm{He}-\mathrm{Ne}$ laser. This laser could be tuned and allowed to generate light consisting of the following wavelengths: 543, 594, 605,612 , and $633 \mathrm{~nm}$. The light passes through a grey filter to obtain the desired intensity. The Glan-Thomson polarizer is a birefringent crystal (calcite) that enables one to obtain highly polarized light. The polarized light passes through the sample placed in a cuvette in the coil connected to a power supply. The state of the polarization of the light (all three Stokes coefficients) was measured in the polarimeter (Thorlab PAX). The measurements were done automatically using LabVIEW. The dependences of the rotation of the polarization plane of the magnetic field (up to $0.4 \mathrm{~T}$ ) were registered for the solutions of nanoparticles as well as for pure solvent. The measurements of the ellipticity were conducted simultaneously.

\section{Results and discussion}

TEM images are shown in Fig. 1a and b. On the basis of these images histograms were obtained, see Fig. $2 \mathrm{a}-\mathrm{d}$. The image analysis allowed us to determine the average diameter of the nanoparticles and the diameter of the metallic core. Respectively, for sample 1 it was $7.1 \pm 1.6 \mathrm{~nm}$ and $5.0 \pm 1.1 \mathrm{~nm}$, and for sample 2 it was $10.9 \pm 1.3 \mathrm{~nm}$ and $6.3 \pm 1.1 \mathrm{~nm}$. The estimated value of the thickness of the oxide shell was $1.1 \pm 0.7 \mathrm{~nm}$ for sample 1 and $2.3 \pm 0.6 \mathrm{~nm}$ for sample 2 .

The best signal for the Faraday effect and the ellipticity measurements was registered for the green laser (543 nm), which is the consequence of (2). The magnetic field was calculated using the calibration curve, and then the curves expressed by (1) were fitted, see Fig. 3. One of the fitting parameters was the magnetic momentum of a single nanoparticle $\mu$. Knowing the bulk magnetic momentum per single atom of cobalt, i.e., $1.72 \mu_{\mathrm{B}}[10]$ ( $\mu_{\mathrm{B}}$ - the Bohr magneton), we were able to estimate both the number of atoms in a single nanoparticle and the diameter of a nanoparticle. The results of TEM, the Faraday effect, and the ellipticity analysis are shown in Table I.

The results of the optical measurements (the ellipticity and the Faraday effect) and statistical (TEM) measurements of the metallic unoxidised core are in coincidence in the case of sample 2 . The darker region in the nanoparticles in the TEM images indicates the metallic core and the lighter one indicates the oxide core (the electron beam is transmitted more effectively through oxide than through bulk metal) [11]. 

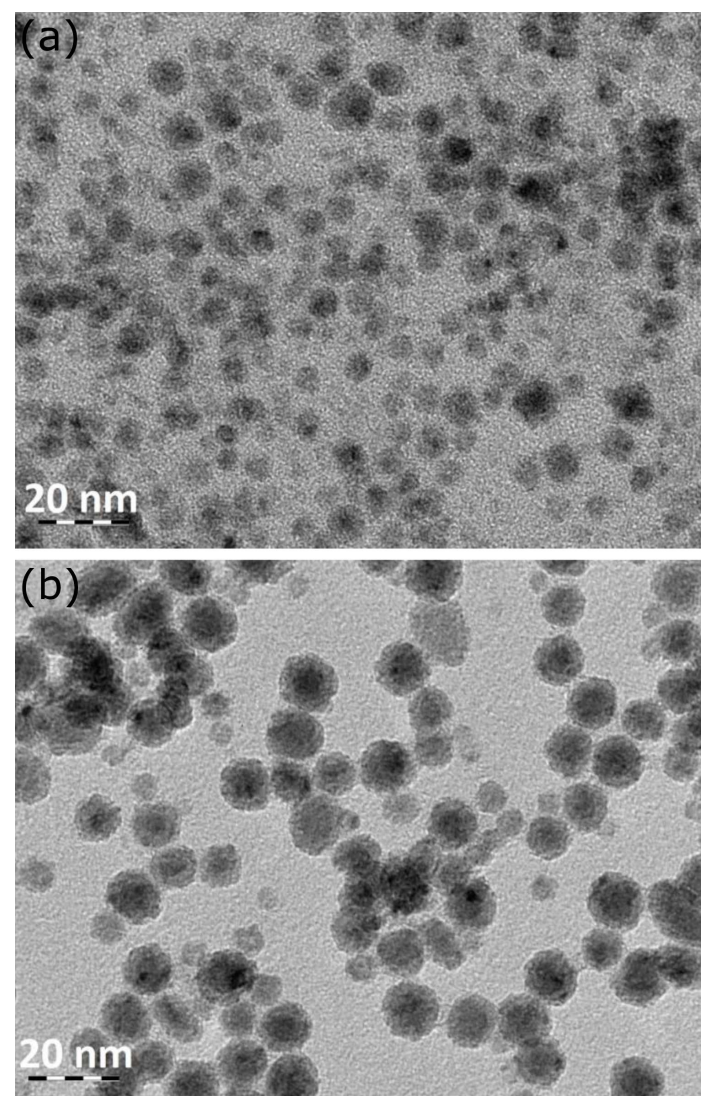

Fig. 1. (a) TEM image of the sample 1, (b) TEM image of the sample 2 .

TABLE I

The results of the statistical analysis of the TEM images and of the measurements of the Faraday effect.

\begin{tabular}{|c|c|c|}
\hline & Sample 1 & Sample 2 \\
\hline $\begin{array}{l}\text { size of the } \\
\text { nanoparticle [nm] (TEM) }\end{array}$ & $7.1 \pm 1.6$ & $10.9 \pm 1.3$ \\
\hline size of the core (TEM) [nm] & $5.0 \pm 1.1$ & $6.3 \pm 1.1$ \\
\hline $\begin{array}{l}\text { magnetic momentum }\left[\mu_{\mathrm{B}}\right] \\
\text { (the Faraday effect) }\end{array}$ & $16500 \pm 2500$ & $25600 \pm 4800$ \\
\hline $\begin{array}{l}\text { calculated size }[\mathrm{nm}] \text { of } \\
\text { the magnetic core }\end{array}$ & $5.8 \pm 0.9$ & $6.7 \pm 1.3$ \\
\hline $\begin{array}{l}\text { magnetic momentum }\left[\mu_{\mathrm{B}}\right] \\
\text { (the ellipticity measurements) }\end{array}$ & $16700 \pm 500$ & $30000 \pm 500$ \\
\hline $\begin{array}{l}\text { calculated size }[\mathrm{nm}] \\
\text { of the magnetic core }\end{array}$ & $5.8 \pm 0.2$ & $7.1 \pm 0.1$ \\
\hline
\end{tabular}

We estimated thickness of the shell by comparing the size of the metallic core obtained by the Faraday effect measurements and the sizes of nanoparticles obtained with TEM analysis (Table I). Due to the higher size of the magnetic core obtained in the Faraday effect, the thickness obtained in this way is smaller. Importantly, the thickness of the shells of the nanoparticles in sample 2 is roughly twice (or three times) greater than that in sample 1.
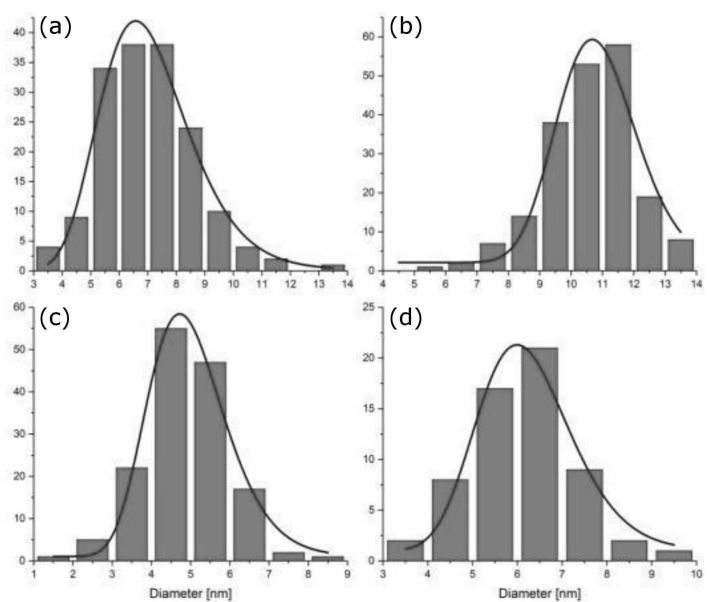

Fig. 2. Histograms of the diameters of nanoparticles obtained for (a) sample 1, and (b) sample 2. Histograms of the diameters of the dark cores of nanoparticles obtained for (c) sample 1 and (d) sample 2. For all of the histograms log-normal distribution curves were fitted (black line). The histograms were made for: (a) 164 , (b) 200, (c) 150, and (d) 60 nanoparticles.
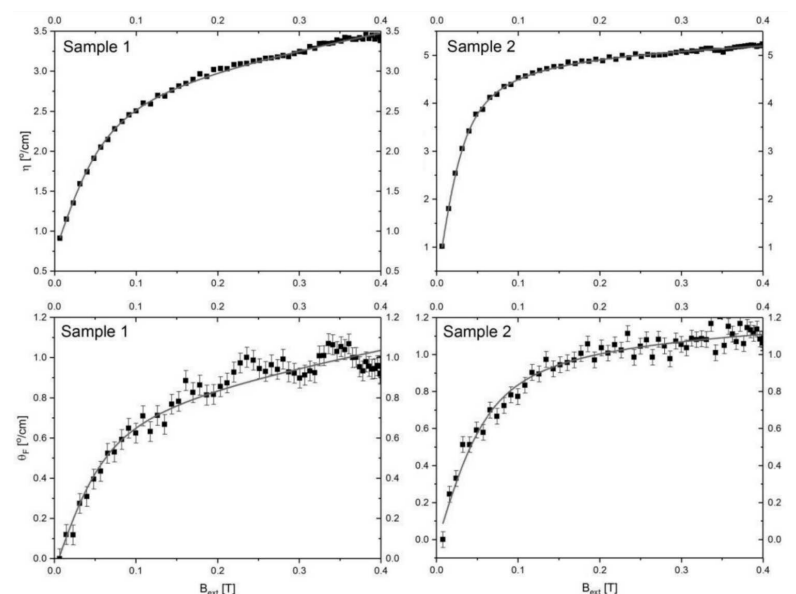

Fig. 3. The dependence of the Faraday angle (top part) and the dependence of the ellipticity (bottom part) as a function of the magnetic field, obtained for both sample 1 and 2, with laser wavelength $543 \mathrm{~nm}$. The theoretical curves were fitted according to (1) and (4). The major fitting parameter was magnetic momentum of a nanoparticle $\mu$.

The stability of sample 2 was higher than the stability of sample 1. The suspensions of the nanoparticles were kept in a few different hermetically closed containers under nitrogen atmosphere. Sample 1 precipitated spontaneously in several jars after $2-3$ weeks, while sample $2-$ after 2 months. It was described previously in the literature that a smooth oxidation of the cobalt nanoparticles conducted after the synthesis, provides higher stability of the nanoparticles [6]. Other authors provide a method to control the thickness of the layer of oxide by adjusting the partial pressure of oxygen [12]. 


\section{Conclusions}

The Faraday effect is a valuable method giving an insight into the diameter of the core of magnetic nanoparticles. In this work we introduced the measurements of the ellipticity as a more accurate method to attain this target. The induced ellipticity is less susceptible to random oscillations in suspensions and is easier to measure having only a small signal reaching the detector. The set shown in Fig. 1-2 is easy to assemble. Moreover, the measurements could be conducted and analysed automatically using programs like LabVIEW. Unlike other methods (TEM, SAXS - small angle X-ray scattering) there is a possibility to apply our method in situ to observe the growth of the nanoparticles during a synthesis.

\section{Acknowledgments}

This work was supported by the National Science Centre Poland (No. 2016/23/B/ST3/03926). All measurements were conducted at University of Warsaw, Poland.

\section{References}

[1] Q.A. Panhkhurst, J. Conolly, S.K. Jones, J. Dobson, J. Appl. Phys. D 36, R167 (2003).

[2] A.-H. Lu, W. Schmidt, N. Matoussevitch, H. Bonnermann, B. Spliethoff, B. Tesche, E. Bill, W. Kiefer, F. Schuth, Angew. Chem. Int. Ed. 43, 4303 (2004).
[3] J.W. Seo, S.J. Park, K.O. Jang, J. Appl. Phys. 85, 5956 (1999).

[4] A.-H. Lu, E.L. Salabas, F. Schuth, Angew. Chem. Int. Ed. 46, 1222 (2007).

[5] L.M. Liz-Marzan, M. Giersig, P. Mulvaney, Langmuir 12, 4329 (1996).

[6] H. Bonnemann, W. Brijoux, R. Brinkmann, N. Matoussevitch, N. Waldofner, N. Palina, H. Modrow, Inorg. Chim. Acta 350, 617 (2003).

[7] D.L. Peng, K. Sumiyama, T. Hihara, S. Yamamuro, T.J. Konno, Phys. Rev. B 61, 3103 (2000).

[8] J. Szczytko, N. Vaupotic, K. Madrak, P. Sznajder, E. Górecka, Phys. Rev. E 87, 033201 (2013)

[9] H.T. Yang, C.M. Shen, Y.G. Wang, Y.K. Su, T.K. Zang, H.J. Gao, Nanotechnology 15, 70 (2004).

[10] J.P. Bucher, D.C. Douglass, L.A. Bloomfield, Phys. Rev. Lett. 66, 3052 (1991).

[11] A. Vincenzo, R. Pietro, M. Moreno, J. Phys. Chem. $C$ 115, 5140 (2011).

[12] H.-G. Boyen, G. Kastle, K. Zurn, et al., Adv. Function. Mater. 13, 359 (2003). 